\title{
La psychiatrie, une science américaine ?
}

\section{Psychiatry, an american science?}

\author{
T. Haustgen \\ (C) Springer-Verlag France 2011
}

Un philosophe qui méditera d'ici quelques siècles sur l'influence des nations dans les différents domaines du savoir, au tournant des deuxième et troisième millénaires, aura sans doute bien des motifs de s'étonner. N'en déplaise aux mânes de Jean-François Revel [10], il établira le constat de l'aventurisme économique des gouvernants de la superpuissance de l'époque - une Amérique qui n'était plus celle de Tocqueville — à l'origine d'une crise bancaire, puis financière, puis sociale, ayant plongé la planète au bord du gouffre, provoqué la paupérisation des pays développés et menacé la construction européenne. Mais il constatera aussi que, malgré ce séisme, la même « grande démocratie » n'aura pas cessé d'influencer, par sa langue, ses modes de pensée, ses recherches et ses publications, les sciences, les techniques et la médecine du monde entier, y compris une discipline mouvante et au statut incertain comme la psychiatrie.

Comme on ne parlera plus à cette époque d'impérialisme, notre philosophe pensera, en s'appuyant sur les impact factors des revues, que la patrie de la liberté a été à l'origine de contributions déterminantes à cette spécialité, tout au long de son histoire. Quelles ne seront pas, à l'issue d'une brève enquête rétrospective, ses interrogations devant la minceur de ces apports, aussi bien en clinique qu'en thérapeutique, comparés à ceux du vieux continent ! S'il n'appellera plus, comme Nancy Andreasen, à un plan Marshall inversé, il pourra toujours invoquer l'immortel La Fontaine : « Selon que vous serez puissant ou misérable/Les jugements de cour vous rendront blanc ou noir ». Et, en fin de compte, il n'aura peut-être pas tort de se dire, en bon épistémologue, que cette même discipline psychiatrique a peu gagné à passer en deux siècles du statut de fille légitime de la philosophie des Lumières et du romantisme à celui de fille adultérine de l'IRM fonctionnelle, des coefficients kappa et des odds ratios.

T. Haustgen $(\bowtie)$

Centre médicopsychologique, 77, rue Vicor-Hugo,

F-93100 Montreuil, France

e-mail : t-haustgen@epsive.fr

\section{Développement de la clinique}

Ce sombre constat n'enlève rien à la vitalité présente de la psychiatrie américaine. Mais il conduit à émettre quelques doutes sur les capacités d'innovation pour le futur de son « leadership » actuel, revendiqué et assumé. Il relativise aussi l'enthousiasme des jeunes collègues pour lesquels l'histoire de notre spécialité commencerait avec le DSM-III en 1980, époque à laquelle, cessant d'être coupée du reste de la médecine, elle aurait enfin émergé d'un âge obscur et ésotérique.

Clinique et nosographie psychiatriques apparaissent au contraire comme la plus belle réussite du couple francoallemand avant les deux conflits mondiaux. Constituées à peu près dans leur forme définitive à la jonction des XIX ${ }^{\mathrm{e}}$ et $\mathrm{xx}^{\mathrm{e}}$ siècles, elles ont connu leur apogée avec les œuvres, complémentaires et concurrentes, du classificateur Kraepelin et du sémiologiste Chaslin, opérant la synthèse des contributions de Kahlbaum (hébéphrénie et catatonie), des frères ennemis de la Salpêtrière, Baillarger et Falret (troubles de l'humeur), puis de leurs élèves Lasègue, Magnan, Cotard et Séglas (délires). Quant à la mise en ordre du groupe des névroses, elle est due comme on sait à Freud et à Janet. Mais, si le terme de psychose a été forgé en 1845 par un autre Viennois, Feuchtersleben, c'est un Écossais, Cullen, qui est à l'origine de celui de névrose (« neurosis ») en 1769, une génération après les traités sur les « vapeurs » des Londoniens Blackmore (1725) et Cheyne (1733).

Au sens strict, la psychiatrie américaine ne peut revendiquer la paternité que de deux affections mentales : la neurasthénie, décrite en 1869, mais par un non-psychiatre, Beard, et écartée des DSM depuis 1980, quoiqu'elle figure toujours dans la CIM-10 ; l'autisme infantile, isolé en 1943 à partir de seulement 11 cas par Kanner. C'est le seul nom de psychiatre américain figurant dans l'index du DSM-III-R, aux côtés de trois Allemands (Alzheimer, Ganser, Pick), deux Français (Briquet, Gilles de la Tourette), un Autrichien (Heller) et un Russe (Korsakov). Mais cette maladie, à travers ses multiples formes atypiques, non spécifiées, envahissantes et désintégratives, a tellement gonflé qu'elle s'est transformée en « fausse épidémie », de l'aveu même du responsable de la task force du DSM-IV [6]. Dans le vaste champ des troubles de la personnalité, sont seuls en mesure de se prévaloir d'une 
origine américaine les états-limites (borderline personality disorder) de Kernberg (1967) et de Grinker (1968).

Les autres découvertes généralement attribuées à la psychiatrie nord-américaine peuvent être contestées. Le trouble schizoaffectif de Kasanin (dont les critères se sont eux aussi notablement modifiés depuis la description initiale de 1933) trouve son origine en France dans les psychoses associées de Masselon et Courbon (1912-1913), puis dans la schizomanie périodique de Claude et Levy-Valensi (1931), en Allemagne dans l'article testament de Kraepelin sur les manifestations de la folie (1920), remettant en cause sa dichotomie classique. La séparation entre formes uni- et bipolaires de la maladie maniacodépressive a été formalisée par Winokur (1972) plusieurs années après les travaux de l'Allemand Leonhard (1957), du Suisse Angst (1966) et du Suédois Perris (1966). Le trouble bipolaire de type II de Dunner (1976) est déjà décrit dans les publications françaises de Marcé sur la folie circulaire (1862) et de Ritti sur la folie à double forme (1883).

La dénomination de « nouvelles pathologies » à propos de certaines entités du DSM est doublement impropre : ces pathologies ne sont pas apparues à la fin $\mathrm{du} \mathrm{xx}^{\mathrm{e}}$ siècle, et elles avaient été décrites précédemment sous d'autres noms. Si l'on répugne à invoquer Montaigne ou Shakespeare, on peut toujours retrouver le tableau clinique complet de l'attaque de panique de Donald Klein (1962) chez l'Écossais Robert Whytt (1767) et chez le Français Étienne Georget (1821), avant l'attaque d'angoisse de Freud (1895). Si l'on n'a pas envie de se plonger dans le journal de l'Anglais Samuel Pepys, dans la description des affres de Blaise Pascal ou dans celle des terreurs du colonel Chabert, on peut recenser les manifestations du fameux PTSD des combattants du Vietnam dans le tableau de l'hystéroneurasthénie traumatique de Charcot [1]. Pour ce qui concerne les « nouvelles addictions », en tout cas celles aux achats, au jeu et au sexe, les 40 volumes des mémoires du duc de Saint-Simon sont amplement documentés. On frémit du défaut de culture historique de ceux qui considèrent TOC, dysthymie, personnalité multiple et syndrome de fatigue chronique comme de « nouvelles pathologies »...

\section{Innovations thérapeutiques}

La psychiatrie américaine n'a pas non plus joué un rôle pionnier dans la mise en place des techniques de soins qui ont modifié la condition des malades mentaux. Les premières thérapeutiques biologiques ont été découvertes entre 1917 et les années 1930 à Vienne, à Budapest et à Rome, non à Baltimore ou San Francisco.

Le seul psychiatre à avoir reçu le prix Nobel de médecine pour l'une de ces découvertes n'est pas un Américain, mais l'Autrichien Wagner von Jauregg auquel certains associent le neuropsychiatre portugais Egas Moniz (J. Axelrod,
G. Edelman et E. Kandel sont des chercheurs en neurosciences, non des praticiens de la psychiatrie).

La révolution psychopharmacologique de l'aprèsSeconde Guerre mondiale est le fait de laboratoires et de cliniciens français et suisses pour ce qui concerne neuroleptiques et antidépresseurs tricycliques (R. Kuhn), d'un praticien australien (Cade) et d'un praticien danois (Morgens Schou) quant au lithium. C'est certes Nathan Kline qui a publié les premières observations de traitement des psychoses par la réserpine, puis des états dépressifs par l'iproniazide, chef de file des IMAO, mais deux ans après la chlorpromazine dans un cas (1954), la même année que l'imipramine dans l'autre (1957). Quant à la clozapine, elle avait été expérimentée dans les pays germanophones dès les années 1970, avant d'être " redécouverte » près de 20 ans plus tard aux États-Unis. Si l'on en croit Thuillier [12], c'est le chimiste français Ernest Fourneau qui aurait synthétisé en 1910 le premier tranquillisant, très proche du Décontractyl ${ }^{\circledR}$ de l'Américain Berger. Le terme de major tranquilizer a également servi à désigner de manière ambiguë les premiers neuroleptiques aux États-Unis, avant l'introduction du vocable antipsychotic, dysharmonieux et stigmatisant, qui s'est cependant imposé. Ce qui explique peut-être les posologies faramineuses prescrites jusqu'à il y a peu outreAtlantique, au mépris d'effets secondaires neurologiques bien connus des cliniciens européens.

Si l'on passe au vaste domaine des psychothérapies, les thérapies familiales et systémiques sont évidemment tributaires des travaux de Bateson, de même que les TCC le sont des recherches sur le conditionnement de Watson et de Skinner. Mais auraient-elles vu le jour, puis connu leur vogue actuelle, reposant sur l'évaluation, sans les médications psychologiques de Janet et la psychanalyse de Freud ? Cette dernière est maintenant vouée aux gémonies, après avoir été portée aux nues. Mais l'antipsychiatre américain Thomas Szasz lui-même, auprès duquel Michel Onfray apparaît comme un aimable compagnon de Freud, désignait toujours en 1978 la psychanalyse comme le paradigme de la psychothérapie : "À l'époque de la jeunesse de Freud, la psychothérapie était aussi inconnue que l'automobile, la radio ou la télévision. Il était par conséquent impossible à quiconque de pratiquer la psychothérapie. Le fait que l'on puisse le faire aujourd'hui est sans doute la plus grande victoire de Freud. Faut-il s'en féliciter ou le regretter?» [11]. Tout est dit.

En réalité, le seul legs thérapeutique incontestable des États-Unis à la psychiatrie est d'ordre institutionnel et sociothérapique. Le non-restraint et l'open-door $\mathrm{du} \mathrm{XIX}^{\mathrm{e}}$ siècle ne sont pas des inventions américaines, mais britanniques. Toutefois, à l'heure où seules des expériences pilotes isolées se développaient en Europe dans ce domaine, Kennedy présentait en 1963 devant le Congrès un projet ambitieux, inspiré des travaux d'Erving Goffman, alors que le contexte économique différait complètement de celui d'aujourd'hui : fermeture des hôpitaux d'état surpeuplés et coûteux, 
traitement rapide « au sein de leur propre communauté » de la plupart des malades mentaux, ouverture de Community Mental Health Centers, financement de maisons de santé pour les patients ne relevant pas de ces centres par les programmes fédéraux Medicare et Medicaid [4]. À partir de 1981, l'administration Reagan coupe brutalement les subventions au niveau fédéral des CMHC [13]. Quant aux nombreux malades mentaux délinquants peuplant les prisons américaines, il ne semble pas que leur pathologie les préserve de la chaise électrique dans certains états de l'Union, comme l'illustrent diverses affaires récemment médiatisées.

Entre-temps, le modèle américain s'est propagé sur le continent européen, via l'Angleterre et l'Italie. Préservée durant une vingtaine d'années de l'écrémage nosographique et des soins séquentiels discontinus grâce à la mise en place du secteur, la France s'est à son tour alignée sur ce qui est devenu la politique officielle de la vieille Europe en matière de soins psychiatriques. Opération menée avec les meilleures intentions du monde, mais au plus mauvais moment, sans les structures alternatives adéquates et le maillage social du Royaume-Uni. Les enquêtes journalistiques récemment publiées sur la psychiatrie française offrent de nombreuses analogies avec celles qu'on pouvait lire au début des années 1980 sur l'état de la psychiatrie américaine [13]. L'idéologie du struggle for life, importée au pays de Saint Vincent de Paul, y a, comme partout ailleurs, transformé l'open door en revolving door. Pour lutter contre cette forme moderne de l'éternel retour, les praticiens dits encore hospitaliers doivent dorénavant soigner les malades dans la rue et faire du « lobbying » auprès des élus locaux...

\section{Du DSM aux neurosciences}

S'il est un épouvantail immédiatement brandi contre la psychiatrie américaine, c'est bien le DSM. Le concept de critère diagnostique, formalisé par Feighner (1972), dans le prolongement des premières échelles d'évaluation (la BPRS d'Overall et Gorham en 1962), trouve néanmoins sa formulation initiale dans celui de « symptôme de premier rang » du phénoménologiste allemand Kurt Schneider (1950). Cette psychiatrie qui compte et qui mesure, se voulant empirique et « athéorique », superposant des axes diagnostiques multiples, est à l'origine du DSM-III (1980). Il faut bien reconnâ̂tre que les réfutations vengeresses du manuel, publiées jusqu'à nos jours, 30 ans après que le DSM se soit imposé partout, ont quelque chose de ridicule. Visant à faire table rase d'un outil classificatoire en méconnaissant ses axes II à $\mathrm{V}$ ou en lui reprochant de ne pas être un traité de psychopathologie, elles ne font que refléter en miroir la position de ses adorateurs. " Certains l'identifièrent à la Bible, d'autres au protocole des sages de Sion ", écrivait déjà en 1986 Lanteri-Laura [9]. Plus intéressantes, les critiques angloaméricaines du DSM ont porté sur le rôle des groupes de pression, des firmes pharmaceutiques et des compagnies d'assurances [3], sur la fiabilité des catégories isolées au détriment de leur validité [8].

Le DSM-V en projet, dont la parution est prévue pour 2013, ambitionne de devenir un véritable traité, avec des sections étiologiques et thérapeutiques. Allen Frances, qui présida le groupe de travail du DSM-IV (1994), critique sévèrement ses successeurs [6] : confidentialité des groupes de travail, culte du secret, nouveaux diagnostics artificiels, ciblés par l'industrie du médicament (tel le « syndrome de risque psychotique »), inflation des faux-positifs par le flou des limites entre normal et pathologique, risques de dérives médicolégales, remise en cause du système multiaxial. Les recommandations du livre blanc publié en 2002 par D. Kupfer, le nouveau responsable de la task force, préconisent de tabler sur les acquis futurs de l'imagerie cérébrale, de la neurochimie et de la génétique, de développer les examens de laboratoire, de privilégier une approche dimensionnelle sur l'approche catégorielle adoptée jusque-là [7]. Alors que nous ne disposons toujours pas du test biologique ou de l'examen complémentaire permettant de diagnostiquer une dépression ou une schizophrénie, qu'on nous promet pourtant depuis quatre ou cinq décennies, le congrès de l'APA de 2010 a confirmé ces orientations, qui enterrent allégrement l'athéorisme proclamé des débuts. Oubliées les fausses pistes qu'ont inaugurées le test à la dexaméthasone ou la supposée différenciation entre panique et anxiété généralisée par la réactivité thérapeutique ! Nous avons échappé de peu à la mutation de la schizophrénie en neuroemotional integration disorder ou dopamine dysregulation disorder. En dépit de l'invocation rituelle à Kraepelin, beaucoup plus réservé qu'on le croit généralement envers l'anatomopathologie de son époque, c'est une psychiatrie non pas néo-, mais prékraepelinienne qui nous est proposée, celle d'Esquirol et de Griesinger. Ce dernier écrivait en 1845 : " Les maladies mentales sont des maladies du cerveau ». Monomanies à la française (émotions, cognitions, comportements) et « somatisme » germanique : la boucle est bouclée après 150 ans d'errance.

\section{De quelques principes méthodologiques}

Et pourtant! Que cela plaise ou non, il faut bien admettre que la discipline psychiatrique s'est constituée sur des bases philosophiques depuis la fin du XVIII siècle, à l'ombre du De anima d'Aristote, ce premier traité de psychologie cognitive, qui souligne déjà la « difficulté de réellement séparer les facultés ». L'empirisme lui-même est une philosophie, revendiquant une prestigieuse filiation anglaise (Bacon, Locke, Hume) [9]. En ces temps lointains où la psychiatrie française naissante inspirait le Quaker de Philadelphie Benjamin Rush, Daquin intitulait son traité Philosophie de la folie (1791), et les deux ouvrages fondamentaux de Pinel s'appelaient Nosographie philosophique et Traité médicophilosophique (six éditions pour la première, deux pour le second). Le médecin de la Salpêtrière puisait la matière de 
ses écrits dans l'empirisme de Locke, dans le sensualisme de Condillac, mais peut-être surtout dans Rousseau et Cabanis, ses contemporains : les rapports du physique et du moral d'un homme libéré de ses fers par le contrat social. Falret, qui déplorait le manque de « lien philosophique » dans les écrits de son maître Esquirol, croyait, à l'encontre de ce dernier, qu'on ne pouvait, en sémiologie mentale, séparer les âmes de Platon : cognition et thymie, devenues raison et passions, intellect et affects. C'était en somme la victoire posthume de Spinoza sur Descartes.

Falret combattait aussi le dualisme, puisqu'il est le premier psychiatre à formuler en 1864, alors que triomphe la médecine anatomoclinique, une théorie de l'articulation entre un socle organique déficitaire et une reconstruction du psychisme par le délire, fondée sur la " réaction de 1a partie de l'intelligence restée saine sur la partie maladive (...). Cette modification organique, véritable cause première de la maladie mentale, ne produit que l'aptitude à délirer. C'est le travail de l'esprit sur lui-même, mis en mouvement par cette altération organique primitive, qui donne lieu ultérieurement à la production du délire » [5]. Ce schéma causal dialectique, qui permet de dépasser les querelles idéologiques, devait être repris, à des titres divers, par Bleuler, par Clerambault, puis par Henri Ey. On en retrouvera la formulation simpliste dans le biopsychosocial de GL Engel, cet organodynamisme du pauvre. Mais, au début $\mathrm{du} \mathrm{xx}^{\mathrm{e}}$ siècle, la psychiatrie américaine n'avait à proposer, en matière d'étiopathogénie, que le concept de réaction d'Adolf Meyer.

Falret se montrait très réservé à l'égard des statistiques en médecine mentale, "servant fréquemment à introduire dans la science des erreurs d'autant plus nuisibles qu'elles sont accréditées sous la forme mathématique ». Quel intérêt d'effectuer des statistiques dans une discipline encore jeune, dont les classes diagnostiques n'ont pas été clairement définies et limitées ? Ne risque-t-on pas d'aboutir, à partir de prémisses erronées, à des résultats contradictoires, comme Parchappe et Baillarger en 1844 dans l'établissement des causes d'une aliénation mentale encore considérée comme unitaire (morales pour le premier, héréditaires pour le second) ? À peu près en même temps que Falret, Claude Bernard, pionnier dit-on de l'evidence-based medicine actuelle, émettait lui aussi de sérieuses réserves sur la «méthode numérique » de son époque. Dans l'Introduction à l'étude de la médecine expérimentale (1865), il recommandait de " chercher à découvrir des faits nouveaux, au lieu d'essayer de réduire en équations ceux que la science possède ». On croit retrouver les mises en garde de Falret et Claude Bernard dans le cri d'alarme d'Allen Frances, stigmatisant des « enquêtes de terrain » programmées aux ÉtatsUnis pour 2011 et 2012, qui consisteraient à « mettre la charrue avant les bœufs, c'est-à-dire avant la définition d'ensembles de critères utilisables lors de ces enquêtes » [6]. Qui n'aperçoit la faille méthodologique d'associer au nombre et à la quantification, indices supposés de précision, le chevau- chement d'entités démultipliées à plaisir, dont les taux de comorbidité varient du simple au triple selon les études, dans le cas des troubles anxieux par exemple? Les données chiffrées, gages d'exactitude, peuvent-elles s'accommoder de l'incertitude sémantique des conditionnels généralement employés dans les commentaires de ces études : serait, pourrait, semble, paraît, suggère, laisse supposer, etc. ? Pas plus qu'elle ne se confond avec la psychologie pathologique, la psychiatrie n'est pas, ne pourra jamais être la neuroscience pathologique. Pourquoi ? Parce que le rêve de Claude Bernard ne s'est pas réalisé : en dépit de sa technicité croissante, la médecine n'est pas devenue la physiologie pathologique.

Parce que nous savons, au moins depuis Georges Canguilhem (1943), que les concepts de norme, de santé et de guérison (recovery) relèvent, non de la statistique et des examens complémentaires, mais de la subjectivité, de la culture, des habitudes et des capacités d'adaptation au milieu : «En matière de normes biologiques, c'est toujours à l'individu qu'il faut se référer (...). Or, s'il n'a pas paru possible de maintenir la définition de la physiologie comme science du normal, il semble difficile d'admettre qu'il puisse y avoir une science de la maladie, qu'il puisse y avoir une pathologie purement scientifique » [2]. On voit qu'il n'est même pas besoin, pour parvenir à ce constat, de recourir à une quelconque spécificité de la psychiatrie. Puissent nos collègues d'outre-Atlantique ne point l'oublier !

\section{Références}

1. Birmes P, Escande M, Moron P, Schmitt L (2005) L'hystéroneurasthénie traumatique dans les « Leçons du mardi » du Pr Charcot : opposition à la névrose traumatique mais anticipation du trouble de stress post-traumatique ? Ann Med Psychol 163:336-43

2. Canguilhem G $(1943,1966)$ Le normal et le pathologique. Presses universitaires de France, Paris

3. Cooper R (2004) What is wrong with the DSM? Hist Psychiatry 15:5-25

4. Decker HS (2007) How kraepelinian was Kraepelin? How kraepelinian are the neo-Kraepelinians? - from Emil Kraepelin to DSM-III. Hist Psychiatry 18:337-60

5. Falret JP (1864) Des maladies mentales et des asiles d'aliénés. Bailliere JB, Paris

6. Frances A (2010) À propos des 19 propositions du DSM-V : on ouvre la boîte de Pandore ! Lett Psychiatr Fr 194 10-14 (trad fr)

7. Guelfi JD (2010) La classification américaine des troubles mentaux : hier, aujourd'hui et demain. Lett Psychiatr VI(5, suppl 2): 4-9

8. Kirk S, Kutchins H (1998) Aimez-vous le DSM ? Le triomphe de la psychiatrie américaine. Trad fr, Les Empêcheurs de penser en rond, Le Plessis-Robinson

9. Lanteri-Laura G (1986) L'empirisme et la sémiologie psychiatrique. In: La Querelle des diagnostics. Navarin, Paris, pp 151-73

10. Revel JF (2002) L'obsession anti-américaine. Plon, Paris

11. Szasz T $(1978,1981)$ Le mythe de la psychothérapie. Payot, Paris

12. Thuillier J (1981) Les dix ans qui ont changé la folie. Robert Laffont, Paris

13. Tremine T, Cousin FR (1985) La psychiatrie en crise aux USA. Les fous, Reagan et quelques autres... Synapse 11:58-65 